\title{
Does Anxiety Affect Neuropsychological Assessment in College Athletes?
}

\author{
Christopher P. Tomczyk, George Shaver, and Tamerah N. Hunt
}

\begin{abstract}
Clinical Scenario: Anxiety is a mental disorder that affects a large portion of the population and may be problematic when evaluating brain injuries such as concussion. The reliance of cognitive testing in concussion protocols call for the examination of potential cognitive alterations commonly seen in athletes with anxiety. Focused Clinical Question: Does anxiety affect neuropsychological assessments in healthy college athletes? Summary of Key Findings: Three studies were included: 1 crosssectional study and 2 prospective cohort studies. One study examined the effect of a range of psychological issues on concussion baseline testing in college athletes. Another study examined the effect of anxiety on reaction time both before and after sport competition in college-aged athletes. The final study examined the effects of psychosocial issues on reaction time during demanding tasks in college athletes. The first study reported slower simple and complex reaction times in athletes with anxiety. The second study found that athletes with high trait anxiety have slower reaction times both before and after competition. The third study reported that demanding tasks led to increased state anxiety which slowed reaction time. Overall, all 3 studies support the adverse effect anxiety can have on cognitive testing in athletes. Clinical Bottom Line: College athletes who present with anxiety at baseline may be susceptible to decreased performance on neuropsychological assessments. Strength of Recommendation: There is level B evidence that anxiety in healthy college athletes can impact neuropsychological assessments, and level $\mathrm{C}$ evidence that anxiety at baseline concussion assessment impacts neuropsychological testing in college athletes.
\end{abstract}

Keywords: brain concussion, baseline testing, reaction time, state anxiety, trait anxiety

\section{Clinical Scenario}

Anxiety is a mental disorder that affects a large portion of the population, especially adolescents and young adults. ${ }^{1}$ Anxiety is an emotional response to a stimulus (external or internal) and is characterized by a mixture of behavioral, cognitive, and somatic symptoms. ${ }^{2,3}$ In recent years, depression has attracted increased attention, which has led to a surge of concussion-centered research, while anxiety has not received the same attention. The lack of attention to the anxiety-concussion relationship is problematic because of the complexity of concussion assessment and the effects anxiety can have on physical and cognitive performance. ${ }^{3-7}$ Initial research has begun to show that anxiety may have an effect on concussion symptomology, including increased symptom reporting and prolonged symptom resolution. ${ }^{2}$ Although symptom reporting is an important facet of the concussion assessment, it does not address potential cognitive changes associated with concussion. ${ }^{8}$ The concussion management protocol calls for comprehensive neuropsychological assessment, both at baseline and postinjury in order to detect potential cognitive deficiencies. Despite the link between anxiety and cognitive performance, ${ }^{4-7}$ limited research exists examining anxiety and neuropsychological testing within the concussion protocol. ${ }^{5}$ However, the effects of anxiety on various neuropsychological assessments have been

Tomczyk is with the Sport Injury Research Lab, Department of Kinesiology, Michigan State University, East Lansing, MI. Shaveris with the Regents Center for Learning Disorders, Department of Psychology, Georgia Southern University, Statesboro, GA. Huntis with the Department of Health Sciences and Kinesiology, Georgia Southern University, Statesboro, GA. Tomczyk (tomczykc@msu.edu) is corresponding author. examined in healthy athlete populations outside the concussion field. ${ }^{7,8}$ These findings begin to beg the question of whether anxiety screening should be a focused component of the concussion protocol at baseline.

\section{Focused Clinical Question}

Does anxiety affect neuropsychological assessments in healthy college athletes?

\section{Summary of Search, "Best Evidence" Appraised, and Key Findings}

- The literature was searched for studies relating to clinical diagnosis of anxiety, self-reported anxiety, state anxiety, trait anxiety, and neuropsychological assessment. Included studies were limited to level 3 evidence or higher. ${ }^{5-7}$

- Three studies were included: 1 cross-sectional study ${ }^{5}$ and 2 prospective cohort studies. ${ }^{6,7}$

- Bailey et $\mathrm{al}^{5}$ examined college athletes and reported that anxiety significantly correlates with simple $(r=.401)$ and complex $(r=324)$ reaction time portions of a computerized concussion assessment.

- College athletes with heightened anxiety at baseline had poorer performance on reaction times compared with those without anxiety. ${ }^{5}$

- Anxiety at the time of baseline neurocognitive testing may limit an athlete's ability to perform at maximal capacity, which is necessary when assessing cognitive ability. ${ }^{5}$ 
- Ciucurel $^{6}$ examined college-aged athletes both before and after a sport performance and found that high precompetition trait anxiety lead to slower reaction times, while high precompetition state anxiety led to faster reaction times.

- Williams and Andersen ${ }^{7}$ examined central and peripheral vision and reaction time both at baseline and during a demanding task (a task that evoked stress by having the athletes listen to a distraction tape while completing all tasks) in college athletes during preseason.

- There was a significant increase in state anxiety between the baseline and demanding task scenarios, although low effect sizes were reported (0.24). However, this increase in stress was reported to slow the athletes' reaction times during the demanding tasks. ${ }^{7}$

\section{Clinical Bottom Line}

There is low- to moderate-level evidence that college athletes' experience of anxiety (both state and trait) may affect neuropsychological assessments. College athletes who present with anxiety at baseline may be susceptible to decreased performance on neurocognitive assessments, specifically in the domain of reaction time (simple and complex). ${ }^{5-7}$ Only Bailey et $\mathrm{al}^{5}$ specifically looked at the concussion baseline assessment, but Williams and Andersen ${ }^{7}$ examined preseason assessments which align with the timeline when baseline concussion assessment would take place. Also, Ciucurel ${ }^{6}$ specifically examined anxiety in healthy college athletes prior to competition which may offer an accurate representation of the state clinicians will encounter the target population. Continued research is necessary to examine the effectiveness of focused anxiety screening within a standard concussion protocol at baseline. Until such time, clinicians should continue to use a multifaceted approach.

\section{Strength of Recommendation}

There is level B evidence supporting the idea that anxiety can affect reaction time during neurocognitive testing in healthy college athletes, but only level $\mathrm{C}$ evidence that anxiety impacts cognitive components of concussion baseline testing (categorized by the Levels of Evidence, the Centre for Evidence-Based Medicine, 2009). Although due to the nature of the topic, the studies are limited to cohort and cross-sectional designs.

\section{Search Strategy}

\section{Terms Used to Guide Search Strategy}

- Patient/Client Group: College athletes

- Exposure: Diagnosed and/or measured anxiety

- Comparison: College athletes with low anxiety

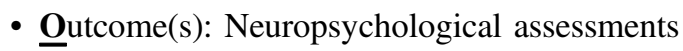

\section{Sources of Evidence Searched}

- MEDLINE/PubMed

- MEDLINE (EBSCO)

- SPORTDiscus Online

- PsychArticles

- ScienceDirect

- Additional resources obtained via review of reference lists and hand search.

\section{Inclusion and Exclusion Criteria}

\section{Inclusion}

- Studies measuring anxiety during baseline concussion assessment in college athletes (ages 18-25).

- Studies measuring anxiety before conducting neuropsychological testing in healthy college athletes.

- Anxiety identified by either a specific measure or by medical diagnosis.

- Anxiety was distinguished from depression and other psychiatric disorders.

- Articles published in English.

- Level 3 evidence or higher.

\section{Exclusion}

- Studies that included athletes with additional concussion comorbidities or pharmacological intervention (ie, learning disorders, ADHD, pharmacological medication).

- Studies that did not include a measure of baseline or precompetition assessment.

- Review articles.

- Not available in English.

- Studies that did not use human subjects.

\section{Results of Search}

Three articles ${ }^{5-7}$ met the inclusion criteria and are presented in Table 1 . Bailey et $\mathrm{al}^{5}$ examined anxiety and the neuropsychological concussion assessment at baseline. Ciucurel ${ }^{6}$ examined anxiety and a neuropsychological assessment before and after sport performance. Williams and Andersen ${ }^{7}$ examined anxiety and a neuropsychological assessment under a demanding task condition. Characteristics of all the articles are presented in Table 2.

\section{Best Evidence}

The studies included best fit the inclusion criteria and examined baseline anxiety in college athletes. There is a clear lack of randomized controlled trial studies centered on the clinical question. However, the methodologies and statistical analyses used were appropriate and led to accurate and strong findings. These studies were selected because they offer initial insight into how anxiety affects neuropsychological testing in the athletic population.

\section{Table 1 Summary of Study Designs of Articles Retrieved}

\begin{tabular}{llcl}
\hline $\begin{array}{l}\text { Level of evidence } \\
\text { (CEBM 2009) }\end{array}$ & Study design & $\begin{array}{l}\text { Number } \\
\text { located }\end{array}$ & Study \\
\hline $3 \mathrm{~b}$ & $\begin{array}{l}\text { Cross-sectional } \\
\text { study design }\end{array}$ & 1 & Bailey et $\mathrm{al}^{5}$ \\
$\mathrm{2b}$ & $\begin{array}{l}\text { Prospective } \\
\text { cohort design }\end{array}$ & 1 & Ciucurel $^{6}$ \\
$\mathrm{2b}$ & $\begin{array}{l}\text { Prospective } \\
\text { cohort design }\end{array}$ & 1 & $\begin{array}{l}\text { Williams and } \\
\text { Andersen }^{7}\end{array}$ \\
\hline
\end{tabular}

Abbreviation: CEBM, Centre for Evidence-Based Medicine. 
Table 2 Characteristics of Included Studies

\begin{tabular}{lll}
\hline Article & ${\text { Bailey et } \mathbf{a l}^{\mathbf{5}}}^{\mathbf{5}}$ & Ciucurel $^{\mathbf{6}}$ \\
\hline $\begin{array}{l}\text { Study design } \\
\text { Participants }\end{array}$ & $\begin{array}{l}\text { Cross-sectional } \\
\text { Participants consisted of 47 D1 football } \\
\text { athletes. The majority of them were in } \\
\text { their first year }(\mathrm{n}=46) \text { and identified as } \\
\text { black (43\%) or white (53\%). The aver- }\end{array}$ & $\begin{array}{l}\text { Prospective cohort } \\
\text { Participants consisted of } 70 \text { male } \\
\text { college-aged athletes participating in judo, } \\
\text { canoe/kayak, swimming, and } \\
\text { tennis. The average age was 20.3 y }\end{array}$ \\
& $\begin{array}{l}\text { age age was 18 y, and all participants } \\
\text { completed the baseline PAI and CRI. }\end{array}$ & $\begin{array}{l}\text { (SD =0.95). Individual-sport athletes were } \\
\text { selected in order to avoid confounding influ- } \\
\text { ence from the relationship between team-sports } \\
\text { athletes and lower anxiety levels. }\end{array}$
\end{tabular}

Intervention investigated

Outcome measure(s)

\section{Main} findings

Level of evidence
Baseline PAI scales/subscales presentations correlation and effect on CRI indexes.

The PAI and CRI were used as a preseason athletic and academic screening, and all data were collected from 2001 to 2003. The PAI consists of 22 distinct scales and subscales (ie, anxiety, depression, suicidality etc).

The CRI is a computerized concussion assessment that measures simple/complex reaction time and processing speed. Primary measures were the significant PAI scales/subscales and the CRI indexes. Three linear regression models were run to estimate effect size of PAI predictors on CRI performance.

The PAI anxiety scale significantly correlated with CRI indexes for simple reaction time $(r=.401)$ and complex reaction time $(r=.324)$.

Significant anxiety subscale correlations for simple reaction time included cognitive $(r=.432)$ and physiological $(r=.308)$ and for complex reaction time included affective $(r=.331)$ and cognitive $(r=.313)$.

Individuals who endorsed higher anxiety symptoms on the PAI performed worse on correlated indices of the CRI (simple and complex reaction time).
The effect of trait and state anxiety on reaction time and motor performance both before and after sports competition.

The STAI was used to measure both trait and state anxiety before and after sport competition. The STAI consists of 2, 20-item questionnaires (S-Anxiety scale and T-Anxiety scale) measure on a 4-point Likert-style scale.

Reaction time was measured using a computerized program that measured simple reaction to the appearance of a visual appearance on the screen.

A motor performance test was used but was unidentified.

Paired samples $t$ tests showed that there was no significant difference in trait anxiety before and after sport competition $(t=4.26, P=.08)$, while significant difference existed in state anxiety before and after competition $(t=16.78, P<.05$, Cohen $d=1.24$ ), where athletes had higher levels of state anxiety before competition. Paired samples $t$ tests demonstrated a significant faster reaction times for the sample $(t=18.21, P<.05$, Cohen $d=-0.40)$ before competition.

Before competition, there was a negative, moderate, and statistically significant correlation between the state anxiety and reaction time $(r=-.53, P<.001)$; along with a direct, moderate, and statistically significant correlation between the trait anxiety and the reaction time $(r=.34, P<.001)$.

After competition, there was a negative, moderate, and statistically significant correlation between the state anxiety and the reaction time ( $r=-.42, P<.05)$; along with a weak, positive, and statistically significant correlation between the trait anxiety and the reaction time $(r=.29, P<.05)$.

$2 b$
Williams and Andersen ${ }^{7}$

Prospective cohort

Participants consisted of 201 intercollege athletes (81 males and 120 females) from 2 NCAA D1 universities, across multiple sports (softball $\mathrm{n}=6$, wrestling $n=7$, football $n=2$, baseball $n=7$, volleyball $n=21$, basketball $n=18$, gymnastics $n=37$, swimming $n=50$, cross country $n=25$, and track and field $\mathrm{n}=28$ ). Participant's ages ranged from 18 to 23 y and were tested during their preseason.

The effect of psychosocial constructs (negative life events, anxiety, etc) on peripheral and central vision and reaction time.

The S-Anxiety scale of the STAI was used to measure the level of state anxiety before and after the demanding task. The reaction time measurement during the demanding task included a peripheral vision task (participants focused on central black dot and pressed a button as soon as they saw a cues), a central vision task (lights would randomly appear in a centralized area, and participants responded by pressing a button whenever a light appeared), and the Stroop test. The Stroop test was performed simultaneously to each vision task while a loud distraction tape played (left ear: loud verbal distraction; right ear: white noise overlaid with color names at 45 words/min).

Paired samples $t$ test revealed a significant increase in state anxiety during the demanding task $(33.9-36.0 ; P<.0001)$ but sported low magnitude of change and effect size (2.1 and 0.24 , respectively).

A series of paired samples $t$ test were run comparing baseline and demanding task condition performance and revealed significant differences for reaction time (in ms; baseline: 349 [58], demanding task 468 [69]; $P<.0001$, ES = 2.05), where athletes had slower reaction times under the demanding task. 
Table 2 (continued)

\begin{tabular}{|c|c|c|c|}
\hline Article & Bailey et al ${ }^{5}$ & Ciucurel $^{6}$ & Williams and Andersen 7 \\
\hline $\begin{array}{l}\text { Validity } \\
\text { score }\end{array}$ & N/A & N/A & N/A \\
\hline Conclusion & $\begin{array}{l}\text { The existence of anxiety can have effect } \\
\text { computerized baseline concussion as- } \\
\text { sessments, specifically the measurement } \\
\text { of simple and complex reaction time. }\end{array}$ & $\begin{array}{l}\text { State and trait anxiety can have a significant } \\
\text { effect on reaction time both before and after } \\
\text { sport performance. Specifically, athletes } \\
\text { presenting with high trait anxiety before } \\
\text { competition experienced slower reaction times, } \\
\text { and athletes presenting with high state anxiety } \\
\text { before competition experienced faster reaction } \\
\text { times. }\end{array}$ & $\begin{array}{l}\text { College athletes have slower reaction } \\
\text { times and increased state anxiety under } \\
\text { demanding task conditions. }\end{array}$ \\
\hline
\end{tabular}

Abbreviations: CRI, Concussion Resolution Index; PAI, Personality Assessment Inventory; STAI, State Trait Anxiety Inventory.

\section{Implications for Practice, Education, and Future Research}

National organizations have recommended a standardized assessment and management protocol for concussion. ${ }^{8}$ These protocols, which often include cognitive, self-report symptomology, and balance assessments, are conducted at baseline to establish the most individualized and accurate premorbid function. The same assessments are then compared postinjury to examine deficits resultant from the concussive injury. ${ }^{8}$ As a result, clinicians rely on baseline assessments to support clinical interpretations. It is important to be aware of any preexisting conditions that could compromise assessment reliability. Conditions such as previous history of concussion and learning disabilities have been examined to influence recovery postconcussion, yet anxiety has not received similar attention. However, anxiety has been shown to affect components of cognition that are important to the baseline assessment. ${ }^{5-7}$

Anxiety is the most common mental disorder in college-aged individuals, with upward of $26 \%$ of undergraduate students experiencing it annually. ${ }^{1}$ Anxiety can have a significant effect on cognitive ability, potentially interfering with baseline neuropsychological testing. ${ }^{4-7}$ Bailey et $\mathrm{al}^{5}$ found that anxiety can hinder concussion baseline testing in college athletes. They reported moderate correlations between anxiety and simple and complex reaction time. Specifically, those athletes experiencing heightened anxiety at the time of baseline testing exhibited poorer performance on both reaction time domains. ${ }^{5}$ Suboptimal baseline performance may lead to an inaccurate representation of normal cognitive functioning, which may lead to inappropriate clinical decisions postinjury. Currently, research examining the effects of anxiety on baseline concussion assessment is extremely limited, but studies conducted in similar athletic contexts can be used to gauge the necessity for further research. ${ }^{6,7}$

Research involving anxiety tends to examine levels of state anxiety and trait anxiety which dictate the frequency, manifestation, and severity of the emotional response to external stimuli. Specifically, state anxiety is the emotional state of the individual depicted by the presentation of symptoms (physiological and psychological) and may fluctuate over time. ${ }^{9}$ On the other hand, trait anxiety is a relatively stable component of personality that predisposes an individual to perceiving external stimuli as stressful or threatening. ${ }^{9}$ It is important to note that these facets often interact to influence the level of anxiety symptoms an athlete is experiencing, which may decrease cognitive performance on baseline concussion assessments.

When college athletes experience high state anxiety, there appears to be an alteration in reaction time, but findings are inconsistent. 6,7 The arousal component of state anxiety may be the cause of the contrasting findings, for it has been shown to have a facilitative effect on physical tasks and a debilitative effect on mental tasks. ${ }^{3,4}$ Specifically, Williams and Andersen ${ }^{7}$ put college athletes through a demanding mental task that increased state anxiety and found that they performed reaction time tasks significantly more slowly. ${ }^{7}$ In comparison, Ciucurel $^{6}$ found that college athletes with high state anxiety had significantly faster reaction times both before and after sport competitions. However, Ciucurel ${ }^{6}$ also found that college athletes with high trait anxiety had significantly slower reaction times both before and after competition. ${ }^{6}$ These contradictory findings begin to outline the complex relationship anxiety has with reaction time. This is important when considering in neuropsychological assessments reaction time is used as index of processing speed. ${ }^{10}$

Even though these studies were not specifically examining baseline testing, they do partially support the findings of Bailey et $\mathrm{al}^{5}$ that athletes presenting with anxiety may be at risk of altered cognitive performance on baseline neuropsychological testing. However, the neuropsychological batteries used by clinicians during concussion evaluation are multifaceted, and test an array of cognitive functions including concentration, memory, attention, and information processing speed. ${ }^{8}$ The neuropsychological battery used by Bailey et $\mathrm{al}^{5}$ (Concussion Resolution Index) contains only reaction time and speed-decision making subtests and does not account for all cognitive components commonly tested in concussion assessment.

Recent concussion research has evolved to examine the development of psychological changes postinjury. Anecdotally, clinicians have seen a rise in postconcussion psychological and mood disorders, but research supporting these claims is scarce. A critically appraised topic by Hixson et al $^{11}$ reported that preinjury state and trait anxiety are not independently associated with an increase in postinjury state anxiety symptoms. Instead, preinjury depression is the strongest predictor of postinjury anxiety, although higher anxiety sensitivity (definition can be found at Hixson et al $^{11}$ ), psychological distress, and symptom scores have been found following concussion. ${ }^{11}$ Assessing these findings in conjunction with the current literature appraisal, it provides some evidence that anxiety has an impact on various aspects of concussion. Measuring anxiety levels at baseline and monitoring them throughout recovery can be beneficial to gain a more thorough understanding of how athletes may perform on concussion neuropsychological batteries assessments. However, more research needs to done in order to make strong evidence-based practice recommendations.

Examining anxiety and how it affects baseline concussion assessment is novel, but preliminary studies have shown a need for further investigation. Anxiety may result in reaction time 
performance deficits within the concussion protocol, but more research needs to be conducted using neuropsychological batteries that encompass a wider array of cognitive functioning in order to gain a more complete perspective. ${ }^{8}$ The preliminary research available demonstrates the potential adverse effects of anxiety on neuropsychological batteries, which are common in concussion assessment. Additional research should be conducted to examine the effect anxiety has on both baseline and postinjury concussion testing before making system-wide changes to protocols. Furthermore, research should investigate the incidence of postconcussion anxiety and its relationship to recovery time. The current concussion protocol does not call for anxiety to be examined in a focused and targeted manner, and the current state of research suggests that using the recommended multifaceted assessment remains the most effective approach to concussion assessment. The available literature does not provide sufficient evidence to include anxiety screening at baseline, but clinicians should be aware of the implications that high levels of anxiety during cognitive testing may be detrimental to performance. As more research is published concerning anxiety and concussion assessment, it will be important for clinicians to be aware of how anxiety may influence concussion measures in order to provide the most appropriate and individualized care for their athletes.

\section{References}

1. American College Health Association. American College Health Association-National College Health Assessment II: Reference Group Executive Summary Spring 2017. Hanover, MD: American College Health Association; 2017.

2. Covassin T, Elbin RJ, Beidler E, LaFevor M, Kontos AP. A review of psychological issues that may be associated with a sport-related concussion in youth and collegiate athletes. Sport Exerc Perform Psychol. 2017;6(3):220-229. doi:10.1037/spy0000105

3. Weinberg RS, Gould D. Arousal, stress, and anxiety. In: Foundations of Sport and Exercise Psychology. 6th ed. Human Kinetics; 2015: 75-100.

4. Eysenck MW, Derakshan N, Santos R, Calvo MG. Anxiety and cognitive performance: attentional control theory. Emotion. 2007;7(2): 336-353. PubMed ID: 17516812 doi:10.1037/1528-3542.7.2.336

5. Bailey CM, Samples HL, Broshek DK, Freeman JR, Barth JT. The relationship between psychological distress and baseline sportsrelated concussion testing. Clin J Sport Med. 2010;20(4):272-277. doi:10.1097/JSM.0b013e3181e8f8d8

6. Ciucurel MM. The relationship between anxiety, reaction time, and performance before and after sport competitions. Procedia Soc Behav Sci. 2012;33:885-889. doi:10.1016/j.sbspro.2012.01.249

7. Williams JM, Andersen MB. Psychosocial influences on central and peripheral vision and reaction time during demanding tasks. Behav Med. 1997;22(4):160-167. PubMed ID: 9138624 doi:10.1080/ 08964289.1997.10543549

8. McCrory P, Meeuwisse W, Dvorak J, et al. Consensus statement on concussion in sport - the 5th international conference on concussion in sport held in Berlin, October 2016. Br J Sports Med. 2018;51:838-847.

9. Spielberger CD. Anxiety and behavior. In: Anxiety and Behavior. Academic Press; 1966:3-22.

10. Guest A, Schneider B. Adolescents' extracurricular participation in context: the mediating effects of 394 schools, communities, and identity. Sociol Educ. 2003;76(2):89-109. doi:10.2307/3090271

11. Hixson KM, Allen AN, Williams AS, Valovich McLeod TC. Is state anxiety, trait anxiety, or anxiety sensitivity a clinical predictor of symptoms in those presenting with mild traumatic brain injury or concussion? J Sport Rehabil. 2017;26(6):588-594. PubMed ID: 28182530 doi:10.1123/jsr.2016-0122 